\title{
Association between a national primary care pay-for-performance scheme and suicide rates in England: spatial cohort study
}

Christos Grigoroglou, Luke Munford, Roger T. Webb, Nav Kapur, Tim Doran, Darren M. Ashcroft and Evangelos Kontopantelis

\section{Background}

Pay-for-performance policies aim to improve population health by incentivising improvements in quality of care.

\section{Aims}

To assess the relationship between general practice performance on severe mental illness (SMI) and depression indicators under a national incentivisation scheme and suicide risk in England for the period 2006-2014.

\section{Method}

Longitudinal spatial analysis for 32844 small-area geographical units (lower super output areas, LSOAs), using populationstructure adjusted numbers of suicide as the outcome variable. Negative binomial models were fitted to investigate the relationship between spatially estimated recorded quality of care and suicide risk at the LSOA level. Incidence rate ratios (IRRS) were adjusted for deprivation, social fragmentation, prevalence of depression and SMI as well as other 2011 Census variables.

\section{Results}

No association was found between practice performance on the mental health indicators and suicide incidence in practice localities (IRR=1.000, 95\% Cl 0.998-1.002). IRRs indicated elevated suicide risks linked with area-level social fragmentation (1.030; 95\% Cl 1.027-1.034), deprivation (1.013, 95\% Cl 1.012$1.014)$ and rurality $(1.059,95 \% \mathrm{Cl} 1.027-1.092)$

\section{Conclusions}

Primary care has an important role to play in suicide prevention, but we did not observe a link between practices' higher reported quality of care on incentivised mental health activities and lower suicide rates in the local population. It is likely that effective suicide prevention needs a more concerted, multiagency approach. Better training in suicide prevention for general practitioners is also essential. These findings pertain to the UK but have relevance to other countries considering similar programmes.

\section{Declaration of interest}

None.

\section{Keywords}

Mental health; suicide; quality and outcomes framework; financial incentivisation; pay-for-performance.

\section{Copyright and usage}

(c) The Royal College of Psychiatrists 2018
Suicide is a major public health issue in many countries worldwide, with close to 800000 people per annum taking their own lives globally. ${ }^{1}$ In the UK more than 6000 people die by suicide each year, with tens of thousands more attempting to take their own lives. Primary care physicians have an important potential role in preventing suicide as they are the most common type of healthcare contact for people who experience distress or mental health problems. ${ }^{3}$ Most people who die by suicide have contact with a primary care physician shortly beforehand ${ }^{4-6}$ with the percentage of people seeing their general practitioners (GPs) prior to death varying from 46 to 91\%. ${ }^{4-8}$ Furthermore, primary care physicians' failure to screen patients for depression may contribute to underdiagnosis and undertreatment, as well as failure to detect patients experiencing suicidal ideation. Therefore, improving physician recognition of depression and assessment of a person with suicidal thoughts or behaviour are integral components of suicide prevention. ${ }^{9,10}$ Focusing on initiatives to improve the quality of care in primary care can have a big impact on improving the recognition and management of chronic conditions such as depression and severe mental illness (SMI), and can improve the detection of suicidal ideation, ${ }^{11}$ especially when combined with coordinated care between GPs and mental health specialists across the healthcare system. However, the impact of primary care on population outcomes is limited by the wide variations that exist in the quality of care of providers. ${ }^{12}$ This has led policymakers to link quality of care to provider remuneration in an attempt to reduce variability and to improve quality across health systems. ${ }^{13}$

The quality and outcomes framework (QOF), one of the most costly and comprehensive pay-for-performance schemes of its kind, was introduced in 2004 in the UK to reward high-quality primary care. ${ }^{14}$ The scheme linked up to $25 \%$ of GP income to performance on quality indicators across four domains: clinical, organisational, patient experience and additional services. Varying payments were awarded to practices mainly for the ongoing management of chronic conditions, including depression and SMI, with adjustments made for practice list size and disease prevalence. The scheme was periodically reviewed and quality indicators were modified or removed, while new indicators were occasionally introduced for existing or new conditions. ${ }^{15}$ In the first 3 years of its implementation, the QOF yielded improved results for intermediate outcomes and incentivised processes of care but the rate of improvement was not sustained, ${ }^{16,17}$ and performance in later years was no higher than levels projected from pre-intervention trends. ${ }^{16,17}$ In terms of improved patient outcomes, the evidence is contradictory. ${ }^{18-20}$ For some conditions there is evidence of lower rates of emergency hospital admission, ${ }^{18,21}$ and reductions in inequalities of delivery of care $^{22}$ but there has been no clear improvement in mortality rates in the UK for physical conditions covered by the QOF. ${ }^{20}$ Previous research has also found no association between practice-level performance on QOF indicators and all-cause mortality in the locality. ${ }^{19}$

In terms of mental health, the QOF includes indicators for patients with SMI, requiring practices to monitor treatment, record an up-to-date care plan and to conduct an annual review incorporating physical checks and health promotion advice. For patients with SMI, the introduction of the QOF was associated with increases in the numbers of service interactions and the 
number of reported comorbidities. ${ }^{23}$ From 2006, the QOF also includes depression indicators requiring practices to assess severity using validated assessment instruments, or indicators requiring practices to review patients within a month of diagnosis and make a biopsychosocial assessment based on 16 'themes' with one of these being suicidal ideation. These SMI and depression indicators are used to manage the chronic nature of these conditions but also to identify individuals at risk. However, existing evidence on the effectiveness of QOF mental health indicators is limited.

Suicide is an important outcome measure for assessing the quality of mental health services. Effective suicide prevention strategies need to identify individual-level risk factors but also factors associated with the social environment in which an individual lives, ${ }^{24}$ as recent evidence indicates marked spatial variability in suicide incidence in England. ${ }^{25}$ It is suggested that area of residence can have an impact on levels of social support, but can also increase the likelihood of developing a mental illness, both of which increase suicide risk. ${ }^{26}$ Ecological studies have shown that area-level effects such as deprivation and social fragmentation are associated with the geographical distribution of suicide. ${ }^{27-29}$

Most studies investigating determinants of suicide have examined specialist mental health services and suicide ${ }^{30}$ or suicide in local and/or regional settings. ${ }^{31}$ However, no published studies to date have examined the relationship between performance on QOF mental health indicators and suicide. The purpose of this study was to examine for the first time the relationship between incentivisation of mental health management in primary care and suicide rates. To achieve this aim we applied spatial analysis techniques to assess the association between practice performance on the mental health domain of the QOF and suicide rates for the localities that the practices serve, while controlling for relevant small-area population characteristics. No ethical approval was needed as no patients were involved and all data were publicly available except for the Office for National Statistics (ONS) mortality data. The data used in this study are freely available and the authors are happy to share in an organised and cleaned final dataset.

\section{Method}

\section{Data sources}

Several data sources were accessed to extract information on numbers of suicides, population size estimates, rurality, social fragmentation, deprivation, quality of care, disease prevalence and spatial coordinates at the lower super output area (LSOA) level. LSOAs are small geographical areas containing approximately 1500 residents. From the ONS we obtained data on case counts for suicides and open verdicts $^{32}$ per LSOA of residence by year of death, broken down by gender and 5-year age bands, for the period 1 January 2006 to 31 December 2014. Our population at risk was people aged 20 years or older as we wished to exclude children and adolescents from our analysis because of differences in the remit of mental health services and depression management provided to children, adolescents and adults. ${ }^{33}$ Classification of deaths for the study used the ICD-10 and included all deaths with a final underlying cause recorded as 'intentional self-harm', 'injury/poisoning of undetermined intent and sequelae of intentional self-harm' or 'injury/poisoning of undetermined intent', which follows conventional practice for government suicide statistics in the UK. We included both suicide and open verdicts in our outcome measure and we refer to this measure as suicides throughout the manuscript. Population counts by gender and age group, as well as information on ethnicity were obtained from the 2011 national Census, at the 2011 LSOA.

Area-level deprivation, as measured by the Index of Multiple Deprivation (IMD) 2007, was available for LSOAs as at 2011. The
IMD measures relative levels of deprivation for all the 32844 LSOAs in England on a continuous scale of deprivation where most of the indicators are based on 2005 statistics. Moreover, we obtained data on rural/urban classification and we constructed a measure of social fragmentation based on Congdon's index (derived from 2011 Census data on private renting, single-person households, single adults and population turnover). The index represents neighbourhood-level conditions that affect social resources such as social cohesion and social capital. ${ }^{27}$ Data-sets on prevalence of SMI and depression, practice list size and practice-level mental health quality of care data were obtained from NHS Digital. As $80 \%$ of LSOAs do not contain a general practice we used spatial analysis techniques to estimate healthcare data at the LSOA level from the practice-level data (a detailed description of this methodology is provided in the supplementary Appendix available at https://doi.org/10.1192/bjp.2018.143).

The number of primary care practice 'hubs' (an LSOA with one or more practices) varied between 8372 in the third year of the QOF (2006-07) and 7778 in the final year (2014-15) including for each year over $99 \%$ of all patients registered with primary care in England. ${ }^{34}$ From 2006 to 2007 onwards, all relevant information was available; i.e. number of practices, total list size and prevalence rates for those over 18 years old in the two relevant QOF clinical domains: SMI and depression.

To better capture quality of care in the mental health domain, as measured by the QOF, we calculated population achievement, ${ }^{17,19}$ defined as

$$
P A m h=\sum N_{i} / \sum(D i+E i)
$$

where $N i, D i$ and $E i$ denote the numerator, denominator and exceptions for QOF indicator $i$, respectively. Population achievement was calculated, separately within each financial year, across the full set of depression and SMI outcome and measurement QOF indicators in the mental health clinical domain. The complete list and descriptions of all depression and SMI indicators with information on the years they were active are provided in the supplementary Appendix.

\section{Statistical analysis}

We fitted a negative binomial model with standard errors clustered at the LSOA level for global between-area variability and/or local variability in neighbouring areas to derive and map age and gender adjusted area estimates of suicide. Negative binomial models report incidence rate ratios (IRR) and are suitable for analysing count data when there is presence of overdispersion, as was the case in our data.

We used one set of negative binomial regression models to investigate the relationship between mental health quality of care, as measured by the QOF, and suicide incidence over time. The dependent variable was annual number of suicides between 2006 and 2014, and we investigated the relationship with the mental health quality of care measure, in the same or up to a lag of 3 years. The same sets of covariates were used for all models. We also conducted several sensitivity analyses under different assumptions. There was an excess of zero-event counts in the study dataset; thus, more than $99 \%$ of data points (LSOAs by age group and gender, over time) did not include a single suicide. Therefore, we investigated how a zero-inflated negative binomial model fitted the data. This model may intuitively seem to provide a better fit to the data, but the standard negative binomial model is more appropriate from a theoretical perspective, as every population group is 'at risk' of experiencing suicide. As an additional sensitivity analysis, we aggregated suicide numbers and population estimates 
in four age groups $(20-24,25-49,50-64, \geq 65)$, according to ONS classification, to address any issues of collinearity between age groups. The economic recession of 2008 occurred in the middle of our study period; thus, to isolate the impact of the QOF on suicide risk from the effects of the economic recession, we ran a sensitivity analysis for the period 2009-2014. Furthermore, conventional practice in the UK is to include open verdicts when investigating and reporting suicide rates. However, to facilitate international comparison, we analysed open verdicts and definite suicides separately. Finally, we investigated the extent to which spatial autocorrelation was present in the data (i.e. level of suicide risk in one locality affecting risk in an adjacent locality) by calculating Moran's $I$, which is a measure of spatial autocorrelation.

Stata v14.1 was used for the principal data management and analyses. For both sets of analyses, we used the nbreg command with the exposure option followed by the IRR specification and we used clustered standard errors for our unit of analyses (i.e. 32844 LSOAs) to take into account the correlation of observations within each LSOA.

\section{Results}

Figure 1 represents the variability in incidence of suicide across regions over the study period. The North East and North West regions had the highest overall suicide rates whereas the London region had the lowest overall suicide rates across the period of study. There were 38511 recorded suicides in England during calendar years 2006 to 2014, inclusive. Figure 2 represents the spatial distribution of suicide over the whole time period (aggregated), at the clinical commissioning group level (CCG), a middle-level organisation structure of the National Health Service (NHS). Figure 3 represents the spatial variability of quality of care in the mental health domain across LSOAs at the census year i.e. 2011. Descriptive statistics on suicide numbers, population size estimates, number of practices, census information, deprivation, prevalence of depression and SMI and mental health quality of care across regions (strategic health authorities) for 2011 are reported in Table 1. The highest prevalence for depression and SMI was observed in the North East and London regions respectively, whereas the lowest prevalence for depression and SMI was found in the London and South central regions respectively.
We found no association between mental health quality of care and suicide in our main model (IRR $=1.000,95 \%$ CI 0.998-1.002). There was also no association after lagging the effects of mental health quality of care for up to 3 years. This finding was consistent in all sensitivity analyses, including the analysis limiting the study period to 2009-2014 (post-2008 recession). In the sensitivity analysis that investigated definite suicide deaths, results were similar (IRR $=1.000,95 \%$ CI $0.998-1.002$ ). All results from the sensitivity analyses are reported in the supplementary Appendix.

No evidence of heterogeneity in the association of interest was found, by health region or deprivation score. Median QOF mental health quality of care across regions varied between $80.4 \%$ (East Midlands) to $83.0 \%$ (North East). As expected, variability was much greater at the LSOA level, partly because of the smaller denominators. For example, in the first analysis year (2006-2007) performance varied from 12.9 to $99.2 \%$ (10th and 90th centiles were $71.3 \%$ and $91.3 \%$ respectively), in the middle year (2009-10) performance varied from 14.3 to $95.8 \%$ (10th and 90th centiles were $76.3 \%$ and $87.7 \%$, respectively) and finally for the last year (2014-15) it varied from 28.3 to $94.2 \%$ (10th and 90th centiles were $68.6 \%$ and $82.1 \%$, respectively).

Table 2 presents IRRs for the coefficients from the negative binomial regression models. Across all age groups, the highest risk for suicide was observed for the 40-44 group (1.876, 95\% CI 1.785-1.972) while a bimodal distribution was observed with peaks in the mid years and those over 85 years $(1.551,95 \%$ CI 1.438-1.673) thus, indicating a non-linear relationship of suicide and age. Female gender $(0.295,95 \%$ CI $0.288-0.302)$ and social fragmentation (1.030, 95\% CI 1.027-1.034) were also strong predictors of suicide across all models, followed by deprivation (1.013, 95\% CI $1.012-1.014)$. Rurality (1.059, 95\% CI 1.027-1.092) was also a strong predictor of suicide risk, where inhabitants in rural areas were found to be more likely to die by suicide.

\section{Discussion}

\section{Main findings and interpretation}

After adjusting for key social and demographic characteristics, we found no association between primary care practice performance on incentivised mental health indicators and suicide rates in practice localities. Although depression and SMI are common among

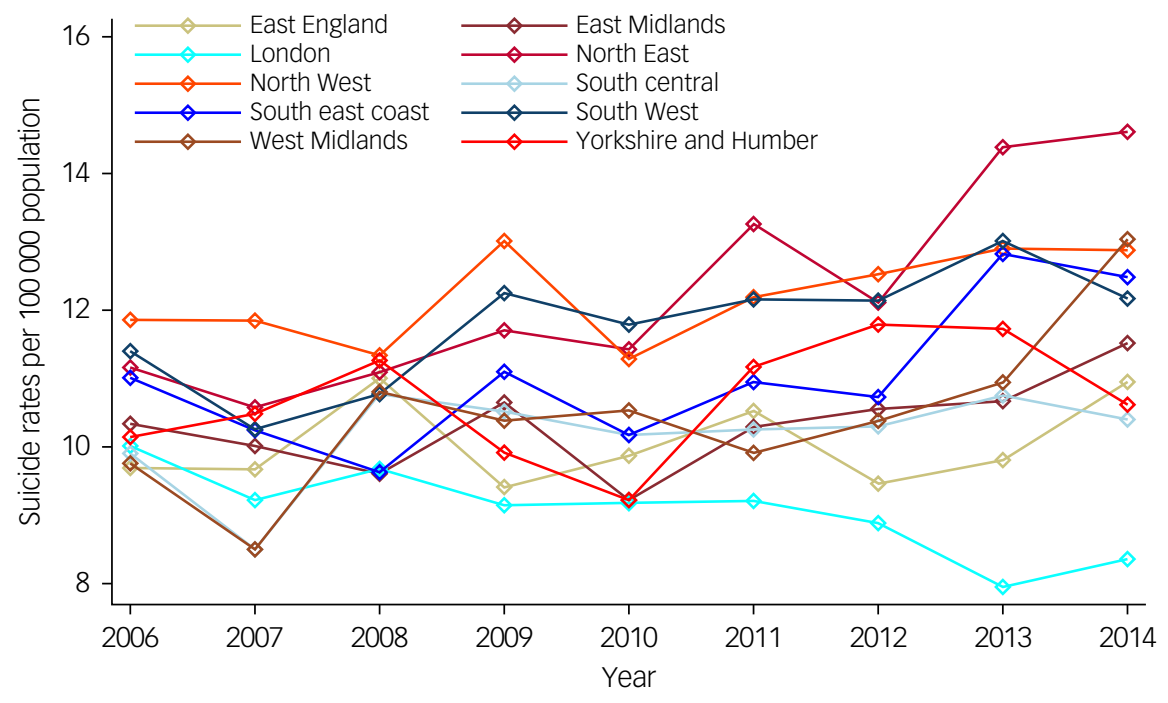

Fig. 1 Suicide rates, per 100000 population, by English regions over time (2006-2014) 


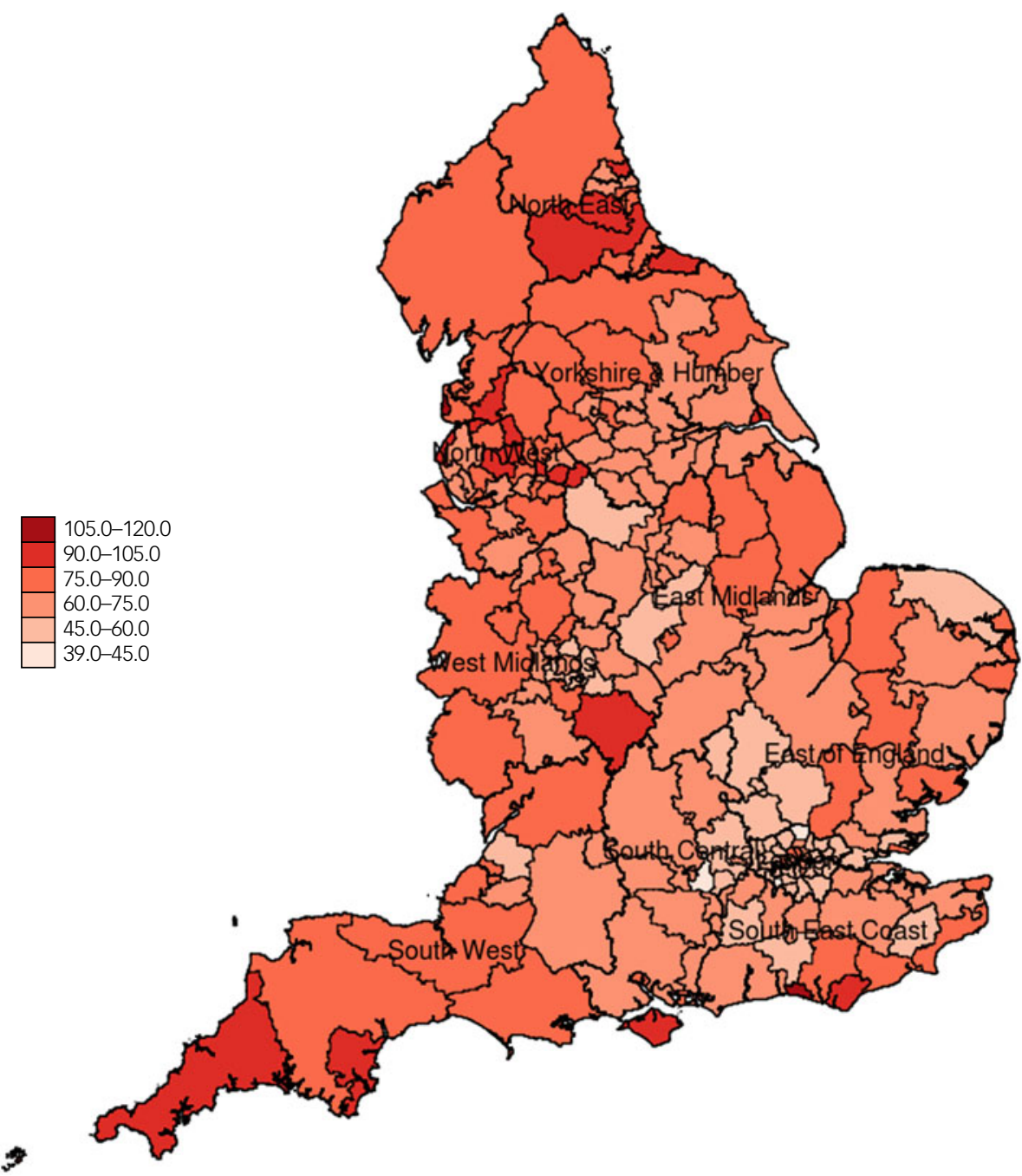

Fig. 2 Suicide rates per 100000 population in England aggregated across all years (2006-2014) and all age groups at the clinical commissioning group level.

people who die by suicide, some people may not experience these symptoms and others may not be diagnosed by their GPs. ${ }^{35}$ Not all people with mental illness see their GP in the year before death occurs $^{4}$ and not all people with mental illness will be assessed or treated. ${ }^{5}$ Nevertheless, GP-based efforts can have positive results in the reduction of suicide rates sometimes associated with an increase in antidepressant prescription. ${ }^{36}$ One of the aims of incentivisation was to achieve better quality of care for people diagnosed with mental illnesses and, although suicide is rare, we might have expected to have observed small effects for a successful programme in that respect. For a dataset of this size, statistical significance is a secondary concern, as we should have been able to identify even a tiny effect - but there appeared to be none. Moreover, QOF mental health 'quality of care' scores do not inform as to care received in settings outside general practice. For example, 28\% of people who die by suicide in the UK have had contact with mental health services in the previous 12 months; ${ }^{37}$ however, as this study focuses on aspects of mental healthcare in general practice we did not account for the important role of mental health and emergency services in preventing suicide. If local mental health services had been consistently beneficial and primary care services poor or vice versa, then the effect of the quality of primary care would be mitigated by the unknown effect of the quality of specialist mental healthcare in the same locality. Finally, even if high quality of mental health provision in primary care, as incentivised through the QOF, has a beneficial impact on patients overall this may not be apparent for important but relatively low frequency markers of population health such as death by suicide.

Our regression models have also indicated that area-level socioeconomic factors, such as social fragmentation and rurality, rather than variations in the quality of primary care, are more strongly associated with suicide risk. We identified a strong relationship between levels of deprivation and suicide for our study period. One additional percentage point in population deprivation is associated with a $1.3 \%(n=52)$ increase in suicides nationally in 1 year. A change in IMD score from the 25th centile (9.57) to the median (17.07) would correspond to an $9.75 \%$ increase in suicide rates $(n=381)$. The extent of the relationship between deprivation and suicide is also highly consistent across the sensitivity analyses reported in the supplementary Appendix. Social fragmentation was a strong predictor of suicide, where one additional percentage point in social fragmentation is linked to $3.0 \%$ increase in suicide $(n=122)$ nationally in 1 year. These findings agree with previous evidence on the effects of deprivation ${ }^{27}$ and social fragmentation ${ }^{27,28}$ and also agree with previous evidence that showed that social fragmentation has a stronger association with suicide risk than deprivation. ${ }^{28}$

Additionally, we observed a moderate association between the prevalence of SMI and suicide across all models and all sensitivity 


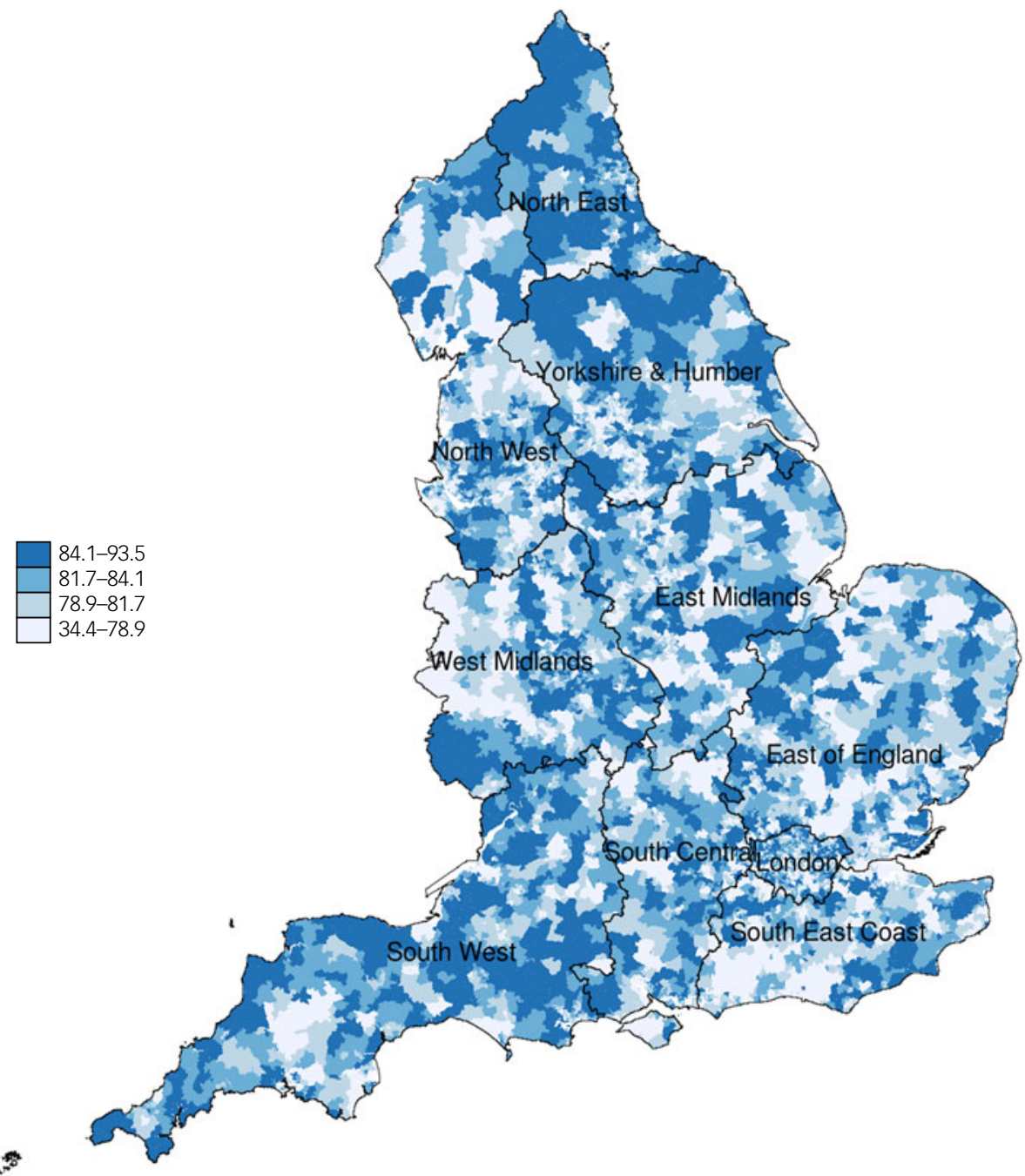

Fig. 3 Measure of mental health quality of primary care in England for specific mental health indicators at the 2011 lower super output areas (2011 Census year). A higher level of the measure in the key represents higher mental health quality of care in the locality.

analyses but small or no associations between prevalence of depression and risk of suicide. As this result may seem unusual we performed a univariate analysis for the associations between prevalence of SMI and depression with risk of suicide. The results from the univariate analysis indicate that prevalence of SMI has a strong effect on suicide (1.481, 95\% CI 1.397-1.570) whereas prevalence of depression has a very small but statistically significant effect (1.005, 95\% CI 1.003-1.008). The fact that unresolved depression register is weakly associated with suicide, despite the well-documented association between depression and suicide, may imply that the condition is not captured well in primary care. Misidentifications and missed cases in depression diagnosis are common in primary care. $^{38}$ Moreover, it is suggested that GP's who work in socioeconomically deprived areas identify the ongoing management of depression as a burden and they may be reluctant to investigate psychosocial issues with their patients because of structural factors and workload. $^{39}$

\section{Strengths and limitations}

This analysis was conducted at the population level and used a novel spatial estimation approach to assess and quantify the relationship between mental health quality of care and suicide rates for the whole primary care-registered population of England. This analysis allows for careful control of population characteristics at a low-level geographical area (LSOA), which is not possible with analyses at the general practice-level. We avoided standardising our outcome variable as standardised death by suicide ratios are imprecise at this low geographical (LSOA) level. Instead we adjusted our analysis for population structure by including all the relevant information on age and gender. This level of analysis was deemed to be optimal as aggregating data at higher geographical levels would introduce heterogeneity and confounding across small-area populations.

However, our study has a number of limitations. First, the possibility of ecological fallacy cannot be ruled out as the associations we observed at small-area level may not pertain to individual patients. Second, in essence we equated performance on the QOF mental health domain to quality of care for mental health, which is not necessarily the case. However, in the absence of other quantifiable metrics, this has become an accepted practice, although the QOF fails to capture other aspects of consultations that may be even more important.

Third, the IMD is by definition an aggregate measure of income, employment, health, education, crime and environmental deprivation in a locality. Thus, it is always collinear with measures of poor health and income, and these were necessarily excluded from our analysis. Fourth, we could not access population measures of ethnicity for all age groups and we included this variable as 


\begin{tabular}{|c|c|c|c|c|c|c|c|c|c|c|}
\hline \multirow[t]{2}{*}{ Aggregates } & \multicolumn{10}{|c|}{ Strategic health authority ${ }^{a}$} \\
\hline & North East & North West & $\begin{array}{l}\text { Yorkshire } \\
\text { and Humber }\end{array}$ & East Midlands & $\begin{array}{l}\text { West } \\
\text { Midlands }\end{array}$ & $\begin{array}{l}\text { East of } \\
\text { England }\end{array}$ & London & South West & $\begin{array}{l}\text { South } \\
\text { central }\end{array}$ & $\begin{array}{c}\text { South east } \\
\text { coast }\end{array}$ \\
\hline Population size & 1998171 & 5365755 & 4011205 & 3461393 & 4217657 & 4465027 & 6195926 & 4107714 & 3158651 & 3415108 \\
\hline Suicide counts ${ }^{b}$ & 265 & 654 & 448 & 356 & 418 & 470 & 571 & 499 & 324 & 374 \\
\hline \multicolumn{11}{|l|}{ Census information, ${ }^{\mathrm{C}}$ medians across 2011 LSOAS } \\
\hline IMD 2007 & 24.8 & 22.3 & 19.8 & 15.7 & 19.8 & 12.3 & 24.4 & 14.7 & 9.4 & 12.0 \\
\hline P10 & 8.2 & 7.2 & 6.9 & 5.5 & 7.0 & 4.5 & 8.8 & 5.7 & 3.1 & 3.8 \\
\hline P25 & 13.7 & 11.8 & 10.9 & 8.8 & 11.3 & 7.4 & 14.5 & 9.2 & 5.1 & 6.9 \\
\hline P75 & 39.7 & 41.0 & 36.8 & 27.5 & 35.9 & 20.2 & 36.0 & 22.7 & 17.7 & 19.8 \\
\hline P90 & 53.2 & 57.6 & 52.1 & 41.5 & 50.5 & 30.2 & 45.6 & 33.4 & 27.1 & 29.7 \\
\hline Social fragmentation & -0.8 & -0.7 & -0.1 & -1.4 & -1.2 & -1.4 & 1.6 & -1.1 & -1.3 & -1.4 \\
\hline P10 & -3.4 & -3.4 & -3.3 & -3.3 & -3.5 & -3.4 & -2.4 & -3.1 & -3.5 & -3.5 \\
\hline P25 & -2.2 & -2.3 & -2.3 & -2.6 & -2.5 & -2.6 & -0.7 & -2.2 & -2.6 & -2.5 \\
\hline P75 & 0.7 & 1.3 & 0.8 & 0.3 & 0.4 & 0.2 & 4.4 & 0.8 & 0.6 & 0.4 \\
\hline $\mathrm{P} 90$ & 3.6 & 4.0 & 3.8 & 3.0 & 2.6 & 2.9 & 6.8 & 4.2 & 3.6 & 4.0 \\
\hline \multicolumn{11}{|l|}{ QOF information: medians across practice hubs } \\
\hline \multicolumn{11}{|l|}{ QOF information (spatially estimated), medians across 2011 LSOAS } \\
\hline Severe mental illness, \% & 0.8 & 0.9 & 0.7 & 0.7 & 0.7 & 0.7 & 0.9 & 0.7 & 0.7 & 0.7 \\
\hline P10 & 0.6 & 0.6 & 0.5 & 0.5 & 0.5 & 0.5 & 0.6 & 0.5 & 0.4 & 0.5 \\
\hline P25 & 0.7 & 0.7 & 0.6 & 0.6 & 0.6 & 0.6 & 0.7 & 0.6 & 0.5 & 0.6 \\
\hline P75 & 0.9 & 1.0 & 0.9 & 0.9 & 0.8 & 0.8 & 1.1 & 0.8 & 0.8 & 0.8 \\
\hline $\mathrm{P} 90$ & 1.0 & 1.2 & 1.0 & 1.1 & 1.0 & 1.0 & 1.4 & 1.0 & 0.9 & 1.1 \\
\hline Depression, \% & 15.0 & 13.5 & 12.1 & 12.6 & 11.4 & 11.5 & 7.9 & 12.6 & 11.8 & 11.3 \\
\hline P10 & 10.9 & 8.9 & 7.0 & 8.2 & 7.7 & 7.0 & 5.1 & 8.9 & 7.7 & 7.7 \\
\hline P25 & 12.8 & 11.2 & 9.2 & 10.1 & 9.5 & 9.1 & 6.4 & 10.6 & 9.6 & 9.5 \\
\hline P75 & 18.6 & 15.5 & 14.4 & 14.6 & 13.6 & 13.9 & 9.6 & 14.8 & 14.0 & 13.4 \\
\hline $\mathrm{P} 90$ & 21.3 & 17.4 & 16.3 & 16.9 & 16.0 & 16.1 & 11.4 & 16.8 & 15.5 & 15.5 \\
\hline$\%$ mental health quality of care $\mathrm{e}^{\mathrm{e}}$ & 83.0 & 81.6 & 81.2 & 80.4 & 82.7 & 80.7 & 81.8 & 82.3 & 82.0 & 80.6 \\
\hline P10 & 78.6 & 76.2 & 75.2 & 74.8 & 77.0 & 74.4 & 76.3 & 75.5 & 75.8 & 74.0 \\
\hline P25 & 80.8 & 79.0 & 78.5 & 77.7 & 80.1 & 78.2 & 79.2 & 79.4 & 79.1 & 77.4 \\
\hline P75 & 84.9 & 84.2 & 83.8 & 83.2 & 84.5 & 83.5 & 84.1 & 84.7 & 84.2 & 83.4 \\
\hline P90 & 86.2 & 86.0 & 85.6 & 85.4 & 86.0 & 85.6 & 85.7 & 86.4 & 85.9 & 85.5 \\
\hline \multicolumn{11}{|l|}{ Other information across LSOAS } \\
\hline Rural, \% & 17.5 & 9.8 & 16.5 & 25.4 & 14.8 & 28.3 & 0.1 & 30.2 & 19.7 & 20.1 \\
\hline \multicolumn{11}{|c|}{$\begin{array}{l}\text { LSOA, lower super output area; P10, P25, P75, P90 are the respective percentiles for each variable. } \\
\text { a. Strategic health authorities were the highest geographical organisational level for NHS England during the study period. This structure was abolished in } 2013 . \\
\text { b. Suicides and open verdicts in } 2011 . \\
\text { c. Data available from the } 2011 \text { census. } \\
\text { d. Quality and outcomes framework (QQF) year } 7 \text { (2010-2011). } \\
\text { e. Mental health quality of care is measured as \% achievement of the population in the relevant QOF indicators. }\end{array}$} \\
\hline
\end{tabular}




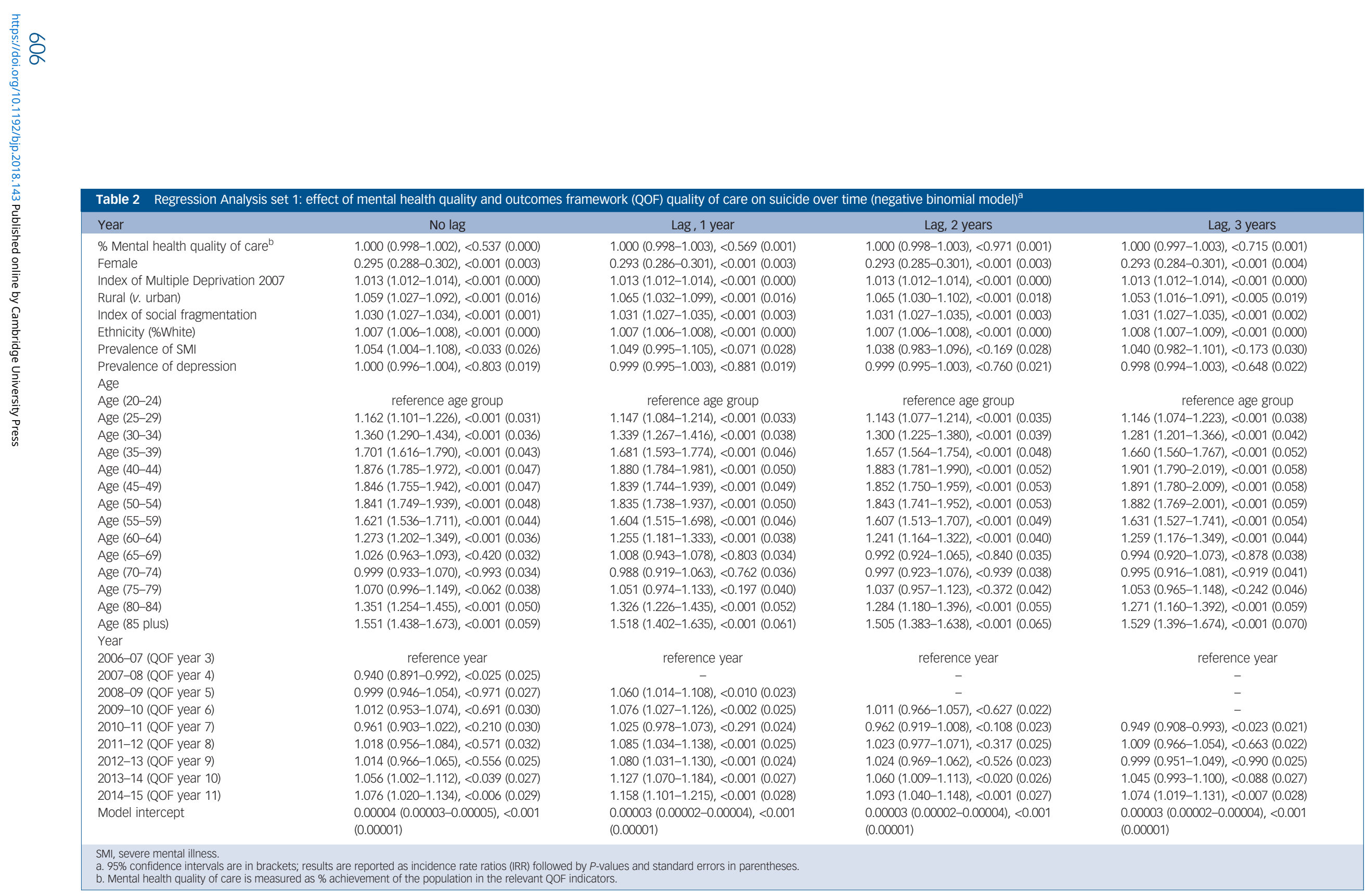

Female

Age $(30-34)$

Age $(75-79)$

2006-07 (QOF year 3)

2007-08 (QOF year 4)

2008-09 (QOF year 5)

b. Mental health quality of care is measured as \% achievement of the population in the relevant QOF indicators. reference age group

nce year

$1.011(0.966-1.057)<0.627(0.022)$ $0.962(0.919-1.008)<0.108(0.023)$ reference age group $1.281(1.201-1.366),<0.001(0.042)$ $1.660(1.560-1.767),<0.001(0.052)$ (1.780-2.019), <0.001 (0.058) $882(1.769-2.001)<0.001(0.059)$ $1.631(1.527-1.741),<0.001(0.054)$ $1.259(1.176-1.349)<0.001(0.044)$ $.271(1.160-1.392),<0.001(0.059)$ reference yea

$0.949(0.908-0.993),<0.023(0.021)$ $1.009(0.966-1.054),<0.663(0.022)$ $0.999(0.951-1.049),<0.990(0.025)$ $1.074(1.019-1.131),<0.007(0.028)$ $0.00003(0.00002-0.00004),<0.001$ $(0.00001)$ 
'\% White' at LSOA level. Fifth, we were unable to attribute practice performance to LSOAs for the first 2 years of the scheme (2004-05 and 2005-06), the period during which practices exhibited the largest improvements in performance, ${ }^{22}$ because of data on mental health quality of care being unavailable. However, there was sufficient variation in quality of care at the LSOA level from 2006-07 to discern any association with suicide risk should it exist.

Sixth, any effects that the scheme might have had on suicide may have been delayed and the time window between mental health quality of care and suicide (i.e. 3-year lag model) could be too narrow. Nonetheless, analysis with longer lag periods is subject to methodological issues, while it seems clinically implausible that the indicators could have long-term preventive effects without short- or medium-term effects. Seventh, some degree of spatial autocorrelation was identified, but considering the very low value of the measure (Moran's $I=0.0049$ ), we did not account for spatial autocorrelation in the model. Even if spatial clustering was present, we would expect it to be driven by socioeconomic factors we controlled for, and not by suicide cases in neighbouring areas, which would warrant the use of spatial regression techniques.

Eighth, multimorbidity and psychosocial risk factors for suicide (for example domestic abuse or substance misuse) may occur concomitantly with depression especially in deprived areas, ${ }^{40}$ however, it is likely that these cases may not be captured adequately in the QOF $^{15}$ and our measure of quality of mental healthcare as some of them will remain unrecorded. Ninth, recording of depression in English primary care has been described as being problematic, as some GPs prefer to record symptoms rather than formal psychiatric diagnostic labels, ${ }^{41}$ but we believe this is unlikely to have affected the validity of our findings in relation to the primary research question. Finally, as death by suicide is used as an underlying indicator in the health and deprivation domain of the IMD, we used the IMD 2007 which was calculated from 2005 data on suicide deaths. Nevertheless, the various IMD versions have been documented to be highly correlated and thus the use of an old version of IMD should not have any implications for our findings.

\section{Suicide prevention}

Our study adds to a growing body of evidence on the potential impact of the QOF on patient outcomes, and is the first study to assess whether quality of primary care, measured as a proxy via metrics generated from a national incentive scheme, is linked with subsequent suicide risk at the population level. Our findings indicate that the scheme appears to have had no impact on suicide risk through the treatment and monitoring of depression and SMI, although population benefits in terms of improved quality of life and reductions in unnatural mortality risk may accrue in the longer term.

Suicide is a complex phenomenon and many factors may influence a person's decision to take their own life. In the absence of indicators related to suicidality and suicidal thoughts and acts, recognition of patients at risk of suicide depends entirely on the GP's ability to identify and interpret relevant signs. This may be extremely difficult without sufficient training as suicidal thoughts may be communicated differently across people at risk. This adds to concerns from GPs regarding the limited training that is provided to them regarding their role in preventing suicide. ${ }^{42}$ Furthermore, the first few weeks following discharge from psychiatric in-patient care are characterised by increased suicide risk for patients ${ }^{37}$ and GPs stress the need to improve communication and information sharing between services, particularly in the cases of hospital discharge and transition between services. ${ }^{5}$ It is vital to establish a collaborative model between GPs, mental health services, community care and hospitals, where improved communication and information sharing will be priorities. However, suicide prevention has never had a higher profile in the UK than at present. The Refreshed Suicide Prevention Strategy, the Health Select Committee Inquiry into Suicide Prevention and the NHS 5-year Forward Review provide a target of a $10 \%$ reduction in the national suicide rate. It is clear that effective suicide prevention requires a more concerted, multiagency approach, including better training in suicide prevention for primary care clinicians.

It is widely recognised that primary care has an important role to play in suicide prevention, but we found no association between practices' reported quality of care on mental health activities incentivised in the QOF and suicide rates in the local population. The QOF was a major national experiment, dwarfing in scale all previous attempts to incentivise high-quality care, but doubts on its impact and value mean that the UK is now retreating from using incentives in primary care, led by Scotland's decision to withdraw from the QOF in 2017. However, other countries continue to use incentives to improve population health, hoping to draw on the experiences of earlier schemes to develop more effective frameworks. If these attempts are to succeed, attention needs to be focused on those activities that contribute to improved patient outcomes, such as reductions in risk of dying prematurely from suicide, and those that will more accurately capture higher value care. More broadly, a better understanding is needed as to how resources can best be allocated to achieve higher quality of services that will lead to genuine improvements in population health outcomes.

\section{Christos Grigoroglou, MA, PhD Student, NIHR School for Primary Care Research, Centre for Primary Care, Division of Population Health, Health Services Research and Primary Care, University of Manchester, Manchester Academic Health Sciences Centre (MAHSC), UK; Luke Munford, PhD, Research Fellow in Health Economics, Centre for Health Economics, Division of Population Health, Health Services Research and Primary Care, University of Manchester, Manchester Academic Health Sciences Centre (MAHSC), UK; Roger T. Webb, PhD, Professor in Mental Health Epidemiology, Centre for Mental Health and Safety, University of Manchester, NHHR Greater Manchester Patient Safety Translational Research Centre, Manchester Academic Health Sciences Centre (MAHSC), UK; Nav Kapur, MBChB, MMedSC, MD, FRCPsych, Professor of Psychiatry and Population Health, Centre for Suicide Prevention, University of Manchester, Greater Manchester Mental Health Trust and NIHR Greater Manchester Patient Safety Translational Research Centre, Manchester Academic Health Sciences Centre (MAHSC), UK; Tim Doran, MBChB, MD, BSC(Hons), MPH, MFPH, Professor of Health Policy, Department of Health Sciences, University of York, UK; Darren M. Ashcroft, BPharm, MSC, PhD, FRPharmS, Professor of Pharmacoepidemiology, Centre for Pharmacoepidemiology and Drug Safety, School of Health Sciences, Faculty of Biology, Medicine and Health, University of Manchester, NIHR Greater Manchester Patient Safety Translational Research Centre, Manchester Academic Health Sciences Centre (MAHSC), UK; \\ Evangelos Kontopantelis, PhD, Professor of Data Science and Health Services Research, Faculty of Biology, Medicine and Health, University of Manchester, Manchester Academic Health Sciences Centre (MAHSC), UK}

Correspondence: Christos Grigoroglou, Williamson Building, University of

Manchester, Oxford Road, Manchester M13 9PL, UK

Email: christos.grigoroglou@postgrad.manchester.ac.uk

First received 18 Jan 2018, final revision 9 Jun 2018, accepted 16 Jun 2018

\section{Funding}

This study is funded by the National Institute for Health Research School for Primary Care Research (NIHR SPCR), through C.G.'S PhD. This report is independent research by the Research (NIHR SPCR), through C.G.'s PhD. This report is independent research by the
National Institute for Health Research. L.M. acknowledges financial support from the MRC Skills Development Fellowship (MR/N015126/1). The views expressed in this publication are those of the authors and necessarily those of the NHS, the National Institute for Health Research, the Department of Health or the Medical Research Council.

\section{Acknowledgements}

We would like to thank the Office of National Statistics and NHS Digital (formerly the Health and Social Care Information Centre) for the wealth of information they have collected and systematically organised, which made this study possible.

\section{Supplementary material}

Supplementary material is available online at https://doi.org/10.1192/bjp.2018.143. 


\section{References}

1 World Health Organization. WHO Suicide Data. WHO, 2017 (http://www.who. int/mental_health/prevention/suicide/suicideprevent/en/).

2 Office for National Statistics. Suicides in the UK: 2015 Registrations. ONS, 2016.

3 Haste F, Charlton J, Jenkins R. Potential for suicide prevention in primary care? An analysis of factors associated with suicide. Br J Gen Pract 1998; 48 : 1759-63.

4 Luoma JB, Martin CE, Pearson JL. Contact with mental health and primary care providers before suicide: a review of the evidence. Am J Psychiatry 2002; 159 909-16.

5 Pearson A, Saini P, Da Cruz D, Miles C, While D, Swinson N, et al. Primary care contact prior to suicide in individuals with mental illness. Br J Gen Pract 2009; 59: $825-32$

6 Andersen UA, Andersen M, Rosholm J, Gram L. Contacts to the health care system prior to suicide: a comprehensive analysis using registers for genera and psychiatric hospital admissions, contacts to general practitioners and practising specialists and drug prescriptions. Acta Psychiatr Scand 2000; 102 126-34.

7 Stanistreet D, Gabbay MB, Jeffrey V, Taylor S. The role of primary care in the prevention of suicide and accidental deaths among young men: an epidemiological study. Br J Gen Pract 2004; 54: 254-8.

8 Windfuhr K, While D, Kapur N, Ashcroft DM, Kontopantelis E, Carr MJ, et al. Suicide risk linked with clinical consultation frequency, psychiatric diagnoses and psychotropic medication prescribing in a national study of primary-care patients. Psychol Med 2016; 46: 3407-17.

9 Goldman LS, Nielsen NH, Champion HC. Awareness, diagnosis, and treatment of depression. J Gen Intern Med 1999; 14: 569-80.

10 Hirschfeld RM, Keller MB, Panico S, Arons BS, Barlow D, Davidoff F, et al. The National Depressive and Manic-Depressive Association consensus statement on the undertreatment of depression. JAMA 1997: 277: 333-40.

11 Mann JJ, Apter A, Bertolote J, Beautrais A, Currier D, Haas A, et al. Suicide prevention strategies - a systematic review. JAMA 2005: 294: 2064-74.

12 Starfield B, Shi L, Macinko J. Contribution of primary care to health systems and health. Milbank Q 2005; 83: 457-502.

13 Van Herck P, De Smedt D, Annemans L, Remmen R, Rosenthal MB, Sermeus W. Systematic review: effects, design choices, and context of pay-for-performance in health care. BMC Health Serv Res 2010; 10: 247-60.

14 Roland M. Linking physicians' pay to the quality of care-a major experiment in the United Kingdom. N Engl J Med 2004; 351: 1448-54.

15 Roland M, Campbell S. Successes and failures of pay for performance in the United Kingdom. N Engl J Med 2014; 370: 1944-9.

16 Campbell SM, Reeves D, Kontopantelis E, Sibbald B, Roland M. Effects of pay for performance on the quality of primary care in England. N Eng/ J Med 2009; 361 368-78.

17 Kontopantelis E, Reeves D, Valderas JM, Campbell S, Doran T. Recorded quality of primary care for patients with diabetes in England before and after the introduction of a financial incentive scheme: a longitudinal observational study BMJ Qual Saf 2013; 22: 53-64.

18 Harrison MJ, Dusheiko M, Sutton M, Gravelle H, Doran T, Roland M. Effect of national primary care pay for performance scheme on emergency hospital admissions for ambulatory care sensitive conditions: controlled longitudinal study. BMJ 2014; 349: g6423.

19 Kontopantelis E, Springate DA, Ashworth M, Webb RT, Buchan IE, Doran T. Investigating the relationship between quality of primary care and premature mortality in England: a spatial whole-population study. BMJ 2015 350: h904.

20 Ryan AM, Krinsky S, Kontopantelis E, Doran T. Long-term evidence for the effect of pay-for-performance in primary care on mortality in the UK: a population study. Lancet 2016; 388: 268-74.

21 Roland M. Does pay-for-performance in primary care save lives? Lancet 2016; 388: $217-8$
22 Doran T, Fullwood C, Kontopantelis E, Reeves D. Effect of financial incentives on inequalities in the delivery of primary clinical care in England: analysis of clinical activity indicators for the quality and outcomes framework. Lancet 2008; 372: 728-36.

23 Kontopantelis E, Olier I, Planner C, Reeves D, Ashcroft DM, Gask L, et al. Primary care consultation rates among people with and without severe mental illness: a UK cohort study using the Clinical Practice Research Datalink. BMJ Open 2015; 5: e008650.

24 Diez Roux AV, Mair C. Neighborhoods and health. Ann N Y Acad Sci 2010; 1186: 125-45.

25 Brock A, Baker A, Griffiths C, Jackson G, Fegan G, Marshall D. Suicide trends and geographical variations in the United Kingdom, 1991-2004. HealthStat Q 2006; 31: 6-22.

26 Middleton N, Whitley E, Frankel S, Dorling D, Sterne J, Gunnell D. Suicide risk in small areas in England and Wales, 1991-1993. Soc Psychiatry Psychiatr Epidemiol 2004; 39: 45-52.

27 Congdon P. Suicide and parasuicide in London: a small-area study. Urban Stud 1996; 33: 137-58

28 Whitley E, Gunnell D, Dorling D, Smith GD. Ecological study of social fragmentation, poverty, and suicide. BMJ 1999; 319: 1034-7.

29 Rezaeian M, Dunn G, St Leger S, Appleby L. The ecological association between suicide rates and indices of deprivation in English local authorities. SOC Psychiatry Psychiatr Epidemiol 2005; 40: 785-91.

30 While D, Bickley H, Roscoe A, Windfuhr K, Rahman S, Shaw J, et al. Implementation of mental health service recommendations in England and Wales and suicide rates, 1997-2006: a cross-sectional and before-and-after observational study. Lancet 2012; 379: 1005-12.

31 Hegerl U, Wittmann M, Arensman E, Van Audenhove C, Bouleau JH, Van de Feltz-Cornelis $C$, et al. The 'European alliance against depression (EAAD)': a multifaceted, community-based action programme against depression and suicidality. World J Biol Psychiatry 2008; 9: 51-8.

32 Linsley KR, Schapira K, Kelly TP. Open verdict v. suicide - importance to research. Br J Psychiatry 2001; 178: 465-8.

33 Department of Health. The National Service Framework for Mental Health Modern Standards and Service Models. Department of Health, 1999.

34 NHS Digital. Quality and Outcomes Framework Achievement, Prevalence and Exceptions Data, 2015/16: Frequently Asked Questions. NHS Digital, 2016.

35 Mitchell AJ, Vaze A, Rao S. Clinical diagnosis of depression in primary care: a meta-analysis. Lancet 2009; 374: 609-19.

36 Rutz W, Vonknorring L, Walinder J. Long-term effects of an educational-program for general-practitioners given by the Swedish-committee-for-the-preventionand-treatment-of-depression. Acta Psychiatr Scand 1992; 85: 83-8.

37 National Confidential Inquiry into Suicide and Homicide by People with Mental Illness. Annual Report, England, Northern Ireland, Scotland and Wales. University of Manchester, 2013

38 Carey M, Jones K, Meadows G, Sanson-Fisher R, D’Este C, Inder K, et al. Accuracy of general practitioner unassisted detection of depression. Aust NZ J Psychiatry 2014; 48: 571-8.

39 Chew-Graham CA, Mullin S, May CR, Hedley S, Cole H. Managing depression in primary care: another example of the inverse care law? Fam Pract 2002; 19: 632-7.

40 Elliott I. Poverty and Mental Health: A Review to Inform the Joseph Rowntree Foundation's Anti-Poverty Strategy. Mental Health Foundation, 2016.

41 Kendrick T, Stuart B, Newell C, Geraghty AWA, Moore M. Changes in rates of recorded depression in English primary care 2003-2013: time trend analyses of effects of the economic recession, and the GP contract quality outcomes framework (QOF). J Affect Disorders 2015; 180: 68-78.

42 Saini P, Windfuhr K, Pearson A, Da Cruz D, Miles C, Cordingley L, et al. Suicide prevention in primary care: general practitioners' views on service availability. BMC Res Notes 2010; 3: 246. 